\title{
Workflow-Management-Systems as enterprise engineering tools
}

\author{
C. Bußler \\ University of Erlangen-Nuernberg, Computer Science Department \\ (Database Systems), Martensstr. 3, 91058 Erlangen, Germany, \\ Tel.+49(9131)8578 84, bussler@informatik.uni-erlangen.de
}

\begin{abstract}
Workflow-Management is a growing research area in computer science. It focuses on the modeling of coarse grain abstract processes called workflows as well as on software architectures and systems for their execution.

Enterprise Integration (EI) deals besides other aspects with enterprise or business processes which are a description of complex tasks within an enterprise involving humans or machines, data and applications. Since business processes developed by techniques of EI should finally be enacted to actively drive the enterprise an execution mechanism is required. We argue that Workflow-Management-Systems (WFMS) are a technology which can be deployed as enterprise engineering tools in order to enact enterprise processes.
\end{abstract}

\section{Keywords}

Workflow-Management, Workflow-Management-System Architecture, Workflow Execution, Business Process Modeling, Enterprise Engineering Tools

\section{ENTERPRISE INTEGRATION AND BUSINESS PROCESSES}

One of the deliverables of EI are guidelines which explain how enterprise entities like humans or machines should or have to work in order to form a more competitive enterprise which operates more successfully on a global market. These guidelines describe different levels of detail: they have to give an idea how each and every enterprise entity behaves as well as how all the entities have to work together and to cooperate in order to achieve a given and to them common goal. A guideline therefore makes goals explicit to all individuals working towards these goals as well as how each of the individual enterprise entities contributes to it.

A means for describing those guidelines are Enterprise Processes or Business Processes. Several authors have shown, how enterprise or business processes are deployed to describe the behavior of an enterprise (see Bussler (1994), Goossenaerts (1993), Graefe (1993), 
Mertins (1993), Scheer (1994), Vernadat (1993)). Vernadat emphasizes the importance of business processes:

"EI is mainly concerned with business integration, $i$. e. the way business processes and enterprise policies are structured and coordinated in the enterprise, how they relate to one another and how they can be efficiently executed using the enterprise means (human resources, applications and physical resources) depending on availability of internal or external enterprise objects (events, information entities, physical entities, etc.) or conditions." (Vernadat(1993)).

It has to be asked, how improved processes can be introduced into the enterprise to improve its behavior, i. e. to change the way of executing business operations in the future. The most appealing approach would be that modeled enterprise processes influence directly the behavior of the enterprise entities in such a way that enterprise entities can rely on some execution mechanism which guides them into the right direction.

The direct change of enterprise behavior through an enterprise model and an execution mechanism is called Model Enactment (Vernadat (1993), Weston (1994)). Several approaches are worked at of how models can be enacted through an execution mechanism which is called Execution Infrastructure or Integration Infrastructure (Katzy (1993), Vernadat (1993), Weston (1994)). These approaches are tailored towards a deployment in a special area of application like factory processes or office processes. Looking from a

more abstract level, these processes have common underlying concepts. This triggers the question: is it possible to provide a common process model together with an execution infrastructure for such a common process model? And, furthermore, can such a combination be deployed in almost all situations where processes are used?

Workflow-Management-Systems (WFMS) address exactly the issue of automatic business process enactment by providing process modeling languages (called workflow modeling languages) which are computationally complete. Therefore modeled processes (or workflows in the context of WFMS) can be processed by an execution infrastructure often called workflow engines. In the remainder of the paper we refer to workflow as a specification of a business process. Even though examples of workflows often refer to business processes in the area of office automation, workflow management does not prescribe concepts from office procedures or any other specific area but provides more abstract concepts from processes in general.

In Chapter 2 (business) process models from the enterprise integration literature are discussed. Additionally, requirements are derived from these process models which have to be fulfilled by WFMS in order to be used in an enterprise on a large scale. Chapter 3 introduces workflow-management-systems from a modeling viewpoint as well as from an information system and software architecture perspective. Chapter 4 discusses how WFMS can be used as enterprise engineering tools and can be deployed as execution infrastructure for business process execution.

\section{PROCESS MODELS IN ENTERPRISE INTEGRATION}

Business process models or enterprise process models (or simply process models) aim to describe the behavior of processes which take place in an enterprise. 


\section{Process Models}

Before discussing the details of several approaches, an overview over the most important underlying concepts is given. To describe a process, several concepts called process aspects are necessary, which can be found in most of the process models discussed later on in this chapter. In addition to the discussion of process aspects a order entry example is modeled:

- Functional Aspect. Processes contain functions to be executed. Examples of such functions are drilling a hole or signing a form. These examples are examples of elementary process steps inasmuch as they are performed by one machine or one human. Processes themselves can group other processes as subprocesses. In case a process has subprocesses it is called a composite process. A composite process can contain process steps as well as other composite processes as subprocesses. Composite processes are used to group or coordinate other processes to achieve a goal which requires several functions to be executed.

- Behavioral Aspect. The behavioral aspect of a composite process defines in which order its subprocesses are executed. The order is specified through control constructs like sequence, conditional branching or parallel branching. Conditional branching depends on one or more predicates which decides dynamically which of the branches is followed.

- Informational Aspect. In general, processes require data as input and produce data as output. E. g. a process step which drills a hole needs the width, depth and precision of the hole to be drilled as input parameters. It maybe delivers an indication of success or failure as output or result parameters. Like to process steps data are passed also to composite processes. They produce data as results, so that there is no distinction between elementary and composite processes. As a side effect of processes material might be moved or transformed. This is reflected in the informational aspect by data referring to the material.

- Organizational Aspect. The organizational aspect of a process is concerned about who (i. e. which machine or human or software service) is responsible for executing a process step. This aspect uses concepts like role or groups to form a structure which is called organization structure. The information captured in an organization structure is used to assign processes to agents (i. e. to determine responsibility).

These process aspects are (according to several authors) at least necessary to model a process such that there is sufficient information about the dynamics of it, see Goossenaerts (1993), Graefe (1993), Mertins (1993), Scheer (1994), Vernadat (1993).

In the following several approaches which can be found in the enterprise integration literature are cited: ARIS (Scheer (1994)), IEM (Mertins (1993)), CIMOSA (Vernadat (1993)), Enterprise Formulae (Goossenaerts (1993)). These are by far not all which can be found in the literature, of course. However, these process models were selected since they are worked on for a long time and are the most representative for their class. The selection is based on the conference proceedings from a series of conferences which were dedicated to enterprise integration or had some special sessions on enterprise integration, see DIISM (1993), ICARCV (1994), IEPM (1993), IMSE (1994), Petrie (1992). A very elaborated evaluation of 11 CIM modeling constructs can be found in CEN TC310 WG1 (1994). 
- ARIS. According to Scheer (1994), the ARIS-approach provides a generic and well documented methodological framework. The ARIS-architecture distinguishes between organization, function, information and control view. Business processes are described by process chain diagrams. The modeling is done using a toolset instead of a language. According to process aspects several subtools are available, each displayed in an own window. The information captured by the ARIS toolset is stored into a database following the ERM (entity-relationship-model). In Scheer (1994) is argued that a formal language imposes restrictions on the day-to-day usability by potential end users. ARIS focuses on the analysis and requirements definition phase during the design of managerial information systems, not on the execution of business processes.

- IEM. The IEM (Integrated Enterprise Modeling) method is based on three classes: product, resource and order. Enterprise data and business processes are assigned to objects of these classes during the modeling. IEM distinguishes between two views: function model and information model. Tasks on objects and business processes belong to functions and so-called linkage-elements like sequence, parallel branching, join and so on. The information model describes the data of an enterprise model based on the three classes mentioned above. Additional to the functional and information model other views can be integrated, e. g. control mechanisms, organization units and costs. How this is done is not explained in Mertins (1993). It is mentioned that a prototype of a modeling tool is under construction. Execution of business processes is not mentioned at all.

- CIMOSA. CIMOSA incorporates an event-driven, process-based modeling approach with the goal to cover essential enterprise aspects in one integrated model. The main aspects are the functional, behavioral, resource, information and organizational aspect. For each of the aspects, modeling constructs are available. This enables to model the aspects of business processes independent from each other. CIMOSA provides a formal language for the modeling, wich is specified in BNF form. Furthermore, CIMOSA aims at the execution of business processes also, not only the modeling of those. The goal is to drive an information infrastructure with the processes modeled.

- Enterprise Formulae. According to Goossenaerts (1993) enterprise formulae are statical mathematical objects which can be developed by simultaneously refining the operationlayer functional requirements of business, engineering and manufacturing processes. An enterprise formulae defines a formal machine, i. e. a dynamic network of formal entities describing interacting government offices, enterprises, persons, machines and their operations. Goossenaerts defines a information infrastructure as a storage-computationcommunication network managing formal machines. Due to its mathematical nature, enterprise formulae are very precise and the goal of enterprise formulae is the modeling as well as the execution of business processes.

\section{Business Processes vs. Workflows}

Originally, process models and workflows had different goals: process models were designed to understand and improve processes in an enterprise by "high level" (i. e. abstract) modeling. Workflow models aimed at providing a way to execute processes in an enterprise. So the question has to be asked why having two kind of models? Why not using the same model for both, business process modeling and execution? To discuss this question, two 
perspectives on business processes are introduced: a business perspective and a information systems perspective. From a business perspective the following issues are important:

- Understanding. One of the most important issues is to understand the processes going on in an enterprise.

- Completeness. Once the processes are descibed it is possible to check, if they are complete or if important operations are left out which might cause operational failures.

- Soundness. As soon as the processes are available on paper, it can be checked if they make sense and contribute to the business goals.

- Necessity. Even though processes are running in an enterprise it is not sure at all that they are necessary. As soon as all processes are registered a necessity check is possible.

- Efficiency. Processes are often not performed in the most efficient way. In most cases this is because nobody can reason about them since they are often not available as models. If processes are captured formally one can figure out where they lack efficiency.

- Improvement. Models of processes allow to reason about improvements which can lead to more efficiency. This in turn improves the overall business.

- Business Rule Conformance. Processes have to be executed according to established business rules. This can be checked if processes are modeled.

From an information system perspective the following issues are of relevance:

- Distribution. If a process is to be executed in several departments of an enterprise, this process has to be available in all systems.

- Transactions. Processes are often transactional, since the output of one step does make sense only in conjunction with the output of others. If one fails, the others have to undo their effects also.

- Reliability. Since processes are mission critical, their execution has to be very reliable.

- Modularity. Developing a process is an investment. If a process can be reused, it saves a lot of money.

- Change Management. Processes change from time to time. Changes to a running system might have a negative impact, if the change is not planned and performed carefully.

- Integration. Processes process data in databases or result in the transformation of material. In order to do this, existing tools and software services have to be used, i. e. integrated to preserve investment and to reuse existing reliable systems.

To summarize, business process models are concerned about how processes help or improve the business to be successful. Workflow models are concerned about the reliable and consistent execution from an information systems perspective. The set of issues in each perspective is very different although both are concerned about the same business processes. 


\section{MODELS AND ARCHITECTURE OF WORKFLOW-MANAGEMENT-SYSTEMS}

As said earlier, if modeled processes could be executed or enacted automatically by an execution infrastructure, the goal of EI could be achieved much more elegantly than without having such a possibility.

In general there are two major possibilities to build an execution infrastructure to execute modeled processes: First, to build an execution infrastructure for a process model from the very beginning. Second, to deploy existing mechanisms which are already available. In the case of processes WFMSs are such existing systems. The first generation of WFMS products are already available, as the huge list of vendors in WfMC (1995) shows. Even though a lot of products are already available, a lot of research has to be done to make these systems more useful for enterprise integration on a large scale. By far not all requirements listed in the previous chapter are fulfilled by existing systems, see Alonso (1994), Georgakapoulos (1994), Jablonski (1994), Marshak (1993). In Section 3.5 one WFMS called MOBILE is discussed.

\subsection{Examples of Workflow Applications}

Applications of WFMS can be found in various publications, see Alonso (1994), Blumenfeld (1993), Breitbart (1993), Jablonski (1995), Medina-Mores (1992), Georgakapoulos (1994). Two of them are described in the following in more detail since they have interesting characteristics, which are very important in the area of enterprise integration. One is the automated execution of business processes in the European Patent Office (Blumenfeld (1993)), the other is the automated execution of business processes in one of the European telecoms (Jablonski (1995)).

\section{European Patent Office}

The European Patent Office (EPO) was founded in 1977 and has 17 countries as members. The benefit of issuing a patent at the EPO instead of at the member countries is that a patent applies in all membership countries. At the moment the EPO employs 3850 employees. 27 million documents are stored, growing about 800000 a year. All documents are categorized into 120000 technical areas. A patent application usually takes $41 / 2$ years for approval. At the time it is issued it comprises 100 pages, after $41 / 2$ years about 220 pages. In the year 1994210000 "active" applications are worked at, i. e. applications which have to be decided on. Each year 60000 applications are filed.

The EPO has implemented its workflows based on a WFMS to speed up the respective business processes. The documents are stored electronically, which makes the access very fast and easy. The EPO deploys a distributed WFMS to handle the huge amount of workflow instances reliably.

\section{A European Telecom}

People move from time to time to a different part of a city or a to different city at all. Since nearly everybody uses telephones, a move means issuing new telephon numbers and making available the old ones for reuse. Of course, people want to keep their old one, so the telephon number moves with them. In addition to that, new telephone lines have to be installed, broken telephones have to be repaired, switches replaced and so on. 
All these customer requests result in the fact that every 2 seconds (!) (in average around the year) a business process is started and has to be executed. Today the company is in the process of deploying a WFMS to handle these requests through automated business processes. Before the automated execution, the average process execution time was 4 weeks. The planned execution time (with the use of a WFMS) is one week.

\section{Conclusion}

The two previous examples make clear that WFMS have to cover a broad range of requirements to be deployed in large enterprises. High volume of workflows is not the exceptional case, but the usual one. Also high throughput has to be possible. The patent office has to act according to law, so all its actions have to be correct. The telecom is doing its business based on the workflows which make sure that the company is earning money. A failure of the WFMS cannot be tolerated by both companies.

\subsection{Requirements for Workflow-Management-Systems}

WFMS are deployed at the heart of an enterprise. Therefore they have to be extremly reliable. In addition to those requirements of Section 2 (distribution, transactions, integration, modularity, change management and reliability) the following requirements have to be fulfilled. It should be emphasized here that not every detail of a process model should be reflected but every detail which is relevant for execution.

- Expressiveness. A WFMS must be able to reflect all details of a process model which is relevant for process execution.

- Scalability. Usually there are lots of processes going on at the same time in an enterprise. Examples are known where the number of processes adds up to several thousands. If processes are automated by an execution infrastructure, a WFMS must be able to scale up to that number while still being peformant.

- Reusability. As known from software engineering, reusability is very important to save development effort as well as to improve reliability. This is also true for process execution. If a process is running without problems, this process is engineered very well. If it is possible, a process should be reused by others like software modules are reused. A typical process step which is reused all the time is a step for signing a document.

- Generality. If the effort is undertaken to build a process execution infrastructure like WFMS, the system should be used in every application where processes are required. In order to be deployed in this way, the infrastructure must be general and tailorable.

- Dynamics. Enterprises have to react to changes every day. It might happen at every single point in time that a process has to be altered to reflect changes. An underlying infrastructure has to be flexible enough to allow this changes to happen dynamically.

- Extensibility. Business processes have several process aspects. However, today it cannot be said for sure that the future does not bring new or different aspects any more. An infrastructure must therefore be able to integrate new process aspects.

Of course, depending on the application area some requirements might not be so important than others. But a general deployable WFMS needs to respond to all requirements. 


\subsection{Workflow Modeling and Execution}

\section{Workflow Modeling}

Workflow modeling languages are used to model workflows which are later on executed. As in modern languages, types and instances are distinguished. Workflow types are modeled and instances of these types are executed. In Section 3.5 a workflow definition language is shown.

The result of a modeling activity is a workflow type which is then compiled and registered. As soon as the workflow type is registered, it can be started, i. e. instances of it can be executed. Of course, several instances of the same workflow type can be executed at the same time. What it means to execute a workflow is discussed in the following.

\section{Workflow Execution}

Workflow execution is straight forward, since principles known from other areas can be applied. We want to discuss how we transferred the way operating system execute operating system processes to workflow execution. Following the operating system principle, a workflow has several states: not existent, ready, running, finished, stopped, and waiting for subworkflows. There is a dispatcher which schedules workflows to be executed and a "processor" which processes workflows. Later on this processor is called kernel. A workflow is always exactly in one state.

State transitions are done during the execution of a workflow. If a workflow is started, it changes its state from not existent to ready. As soon as the kernel has a time slice available, the workflow state is changed to running and the kernel starts executing it. If a subworkflow is started the father workflow waits until the subworkflow is finished. Its state is then waiting for subworkflows. Since the principle is well known not all state transitions are enumerated here. States and state transitions form a complete and defined semantics for executing workflows.

\subsection{Workflow-Management-System Architectures}

Architectures of WFMS are not yet mature enough to discuss their details. Publications are showing only high level architectures so that no real comparison can be provided, see Alonso (1994), Georgakapoulos (1994), McCarthy (1993), Woetzel (1993). However, directions can be observed which we want to mention here. Figure 1 shows the basic architecture of a WFMS. It is high level so that it fits every WFMS. Basically in the center there is a workflow engine which is a server handling the workflow execution. Connected to it is some storage mechanism like databases, which contains workflow type definitions as well as status information from running workflow instances. Clients of an engine are the following interfaces: modeling environments to model workflow types, system management environments to manage the running WFMS as well as worklists for the end users to view and execute assigned steps.

In the next section a WFMS with the name MOBILE is introduced. It can distribute its components over several nodes of a network. Also, it can be connected with others to cooperate (cooperating WFMS). 


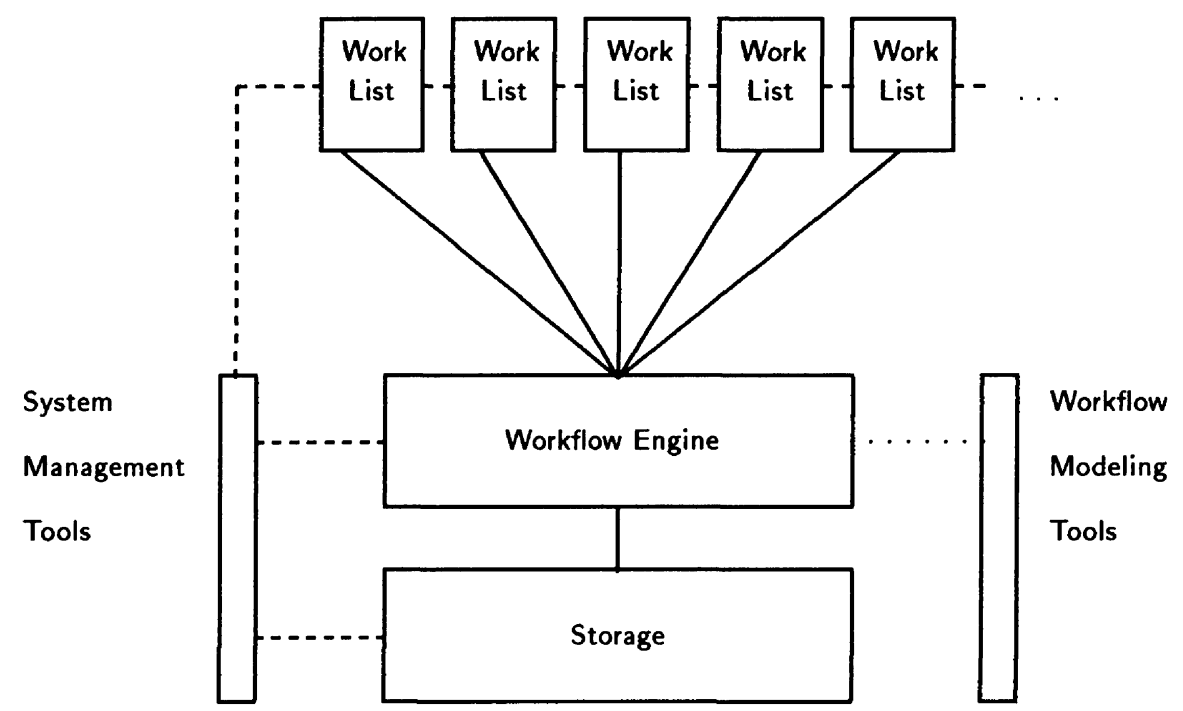

Figure 1 Basic architecture of a WFMS.

\subsection{MOBILE: A Generic Workflow-Management-System}

The approach of MOBILE is to provide an engineering tool, which enables an enterprise to tailor a WFMS to its specific needs (Bussler (1993), Bussler (1994), Bussler (1995), Jablonski (1994), Jablonski (1995), Schuster (1994)). MOBILE follows the idea of providing a definition language, where the objects and concepts to be used for modeling can be built first. This includes not only the informational aspect but also the behavioral as well as the organizational aspect. After the modeling concepts are built themselves, workflow types can be modeled.

\section{MOBILE Modeling Language}

WFMS do not prescribe constructs from certain application area. To support this we model an abstract workflow in the following.

- Data types. Data types are modeled like in conventional programming languages:

TYPE $D_{-} 1$ is Integer;

TYPE D_2 is RECORD a: Integer; b: String; END_RECORD;

- Control flow constructs. Control flow constructs determine the order of workflows. As example sequence, parallel branching, and a join are modeled. To do this we utelize the states and state transition operations from the state transition diagram above $(A$, $B$ and $C$ are workflows):

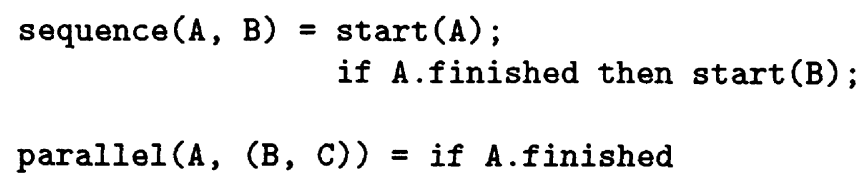




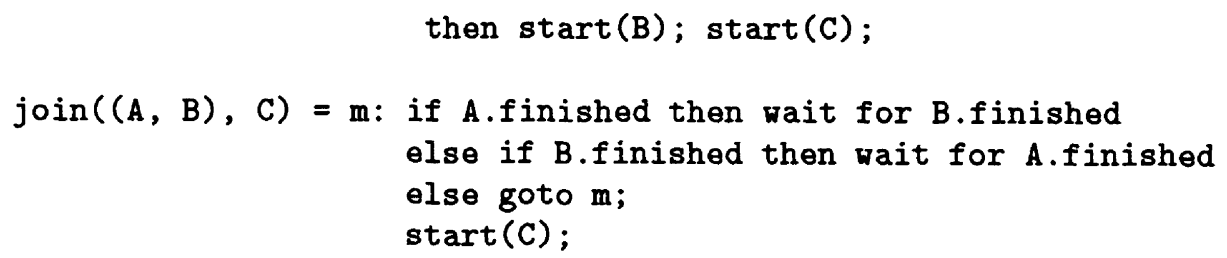

This is the way how control flow constructs can be modeled themselves instead of prescribed by the workflow-management-system.

- Organizational rules. Organizational rules derive agents (users) responsible for a certain workflow to be executed. In short, these rules are predicates over an organization structure deriving to a subset of all agents available. As an example we define the organizational policy $a$ :

$a=$ select all agents which can drill precision holes.

This organizational policy derives to all drilling machines with a very high precision. Policy can become very complex and sophisticated. Bussler (1995) gives several examples.

Workflow types. Before modeling a complex workflow its subworkflows have to be modeled. The workflow types of $A, B, C, D$ and $E$ have to be modeled first to model $F$ below. We assume that these are elementary workflows referencing an application.

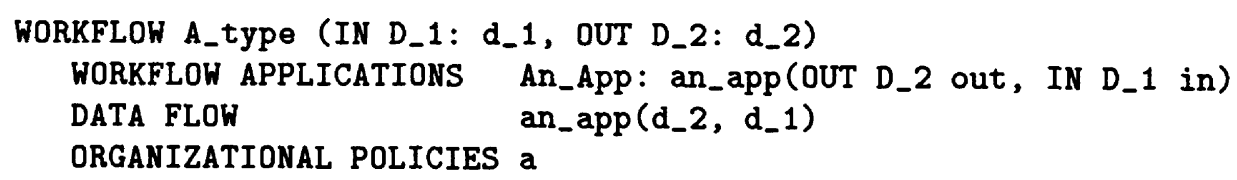

The workflow with the name A_type has one input and one output parameter. An application is called with the input parameter and produces an output which is transferred into the output parameter. The organizational policy is $a$.

We so far modeled all necessary elements for workflow type A_type. The other subworkflows are modeled in a similar way so that their specifications are omitted. Having prepared all these modeling constructs, workflow $F$ can now be modeled:

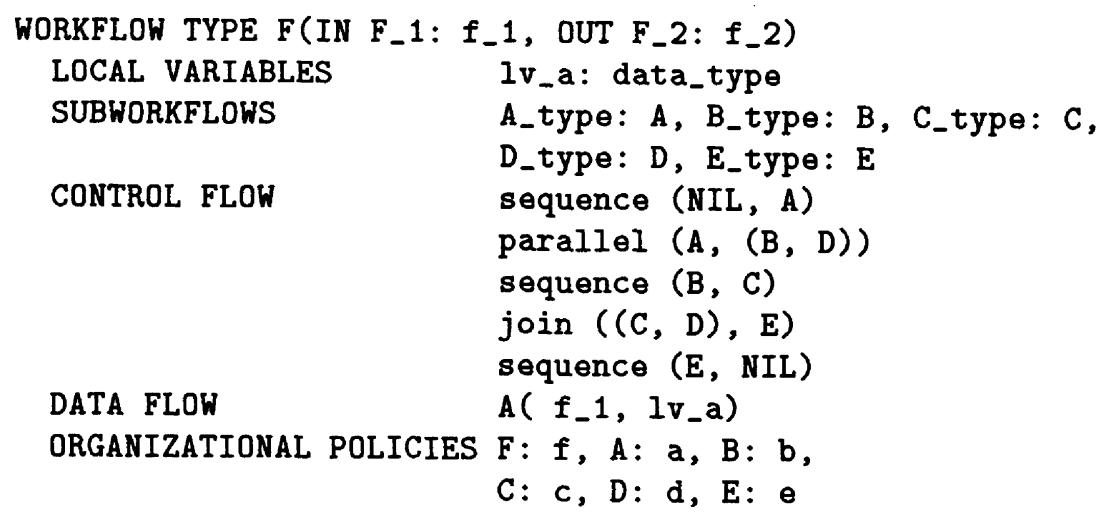




\section{Architecture of the MOBILE System}

The modules MOBILE is composed of are listed in the following. This was decided on in order to have "specialists" for all the necessary functionality. Each of the modules is placed within a server. The servers communicate with each other through messages in a client/server fashion. Since messages can be sent over the net, distribution of the servers is of no problem. The servers are

- Kernel. The kernel implements the functional aspect. It is responsible for the execution of workflows. It instantiates and processes them according to the state transitions discussed previously. During the execution of a workflow the other aspects have to be processed also, which is done by the servers discussed next.

- Control Flow Server. The control flow server implements the behavioral aspect. It takes care of evaluating for a workflow the subworkflows to be executed according to the control flow constructs. During the execution of a workflow the kernel asks the control flow server to evaluate the subworkflows to be started next.

- Data Server. The data flow server takes care of the data of a workflow. This encompassed the data passed between workflows as well as data which are processed by a workflow like CAD drawings or production plans.

- Organization Server. The organization server contains the organization structure as well as the organizational policies. It is asked by the kernel about policies to be evaluated for a workflow as soon as the workflow is ready to be assigned to a user. The organization server returns a set of all users, often only one, who is responsible for execution a workflow.

- Application Server. This server has all application programs registered which might be used by a WFMS. If a user wants to start executing a workflow, the application defined within the workflow specification is started. Since this application is registered within the application server, the user node knows where the application is located and is therefore able to start it.

- User Agent. The users agent is the user interface of users, i. e. the worklist. Each user which works has its own user agent running.

Kernels can communicate with other kernels. This enables the distributed execution of workflow instances. One kernel can ask another one to take over the execution of a workflow instance. All the data with constitute the workflow instance are sent to the remote kernel. This kernel incorporates the data of the workflow and continues processing. This way suborganizations of an enterprise can cooperate easily by sharing the execution of distributed workflows.

\section{DEPLOYMENT OF WORKFLOW-MANAGEMENT-SYSTEMS AS ENTERPRISE ENGINEERING TOOLS}

A proper functioning interplay between process modeling and process execution is essential to make process enactment successful. The automatic execution of processes makes sense only if the modeled processes are correct and only if they can be mapped into an execution infrastructure without loosing modeled details. 


\subsection{Process Modeling and Workflow Definition}

Business processes are modeled as usual using process modeling techniques. Of course, if a technique is similar to the philosophy of the workflow modeling language it eases the transformation later on. However, this is not required.

As soon as a business process is modeled, the elements which are important for execution have to be determined. A good way of doing this is to classify each part of the business process according to the modeling aspects introduced in Section 2. For each of the resulting set a mapping has to be done to the workflow modeling language.

In general, this is not a 1:1 mapping. Elements which have nothing to do with execution do not have to be mapped at all. Others have to be mapped. E. g. an element saying something about the order of two business process steps can be mapped to the control flow construct representing a sequence directly. Some elements might not be mapped directly, e. g. a business process step which is very abstract might be decomposed into several elementary workflows if it turns out that it is not possible for one user to achieve.

Often after the mapping process detailed information is missing to complete a workflow specification. This detail information has to be supplied. This can be some indication that the modeling tasks did not discover all necessary information. On the other hand this is the usual case since e. g. on the level of business process modeling types of data are not important at all. Talking about production plans is sufficient. On the level of workflow specification however the type of a production plan is important for automatic execution.

After the workflow is specified it is available for execution. It therefore can be released. Usually some analysis takes place before a workflow is released and often some simulation is done also. However, we do not want to discuss this here.

\subsection{Workflow Execution and Feedback of Information}

During workflow execution statistical data can be collected. E. g. how often a workflows is started, the average duration of the execution of a workflow, how many failures occurred and so on. This statistical information might be very interesting for business process modelers and people from the administration department. The information can indicate if corresponding business processes need improvement. E. g. if a very important workflow takes a long time to be finished, it might be a good idea to check, if some optimization is possible. In case a lot of failures occur in a workflow, this has to be checked and improved.

The collection of statistical data can therefore leed to feedback to the business process modeling task. Business processes might be remodeled (or "re-designed") in order to be more productive for an enterprise. Of course, the mapping to a workflow specification has to be done again. Otherwise the benefit would not be at the execution level.

\section{SUMMARY}

Enterprise integration needs support from enterprise engineering tools. This papers focuses on the tool support for business processes from the perspective of automatic information system supported execution of business processes in an enterprise. It is shown how business processes modeled by business process modeling techniques from the enterprise integration research community are mapped to execution infrastructures, in this 
case workflow-management-systems (WFMSs). WFMSs provide computationally complete modeling languages for business processes. Once a business process is modeled by a workflow modeling language it can be executed by a WFMS.

The WFMS MOBILE is introduced in detail to show how workflow-managementsystems work. An example of MOBILE's workflow modeling language is shows as well as its architecture. The basic principle of workflow execution is explained also.

Finally a method is laid out how WFMSs can be deployed as enterprise engineering tools. They form a basis on top of business processes modeling can be done. Modeled business processes can later on be transformed into workflows for execution within the enterprise.

\section{REFERENCES}

Alonso, G., Agrawal, D., Abbadi, A., Mohan, C., Günthör, R. and Kamath, M. (1994) Exotica/FMQM: A Persistent Message-Based Architecture for Distributed Workflow Management. IBM Research Report, San Jose, CA, USA.

Blumenfeld, M. (1993) ELFOS: Elektronische Schriftgutverwaltung am Europäischen Patentamt. In: Forschung und Praxis, T 37: IAO-Forum: Dokumenten-Management. Springer-Verlag.

Breitbart, Y., Deacon, A., Schek, H. and Weikum, G. (1993) Merging Application-centric and Data-centric Approaches to Support Transaction-oriented Multi-system Workflows. In: SIGMOD Record, Vol. 22, No. 3.

Bußler, C. and Jablonski, S. (1993) Process Modeling and Execution in Workflow Management Systems. In: Proceedings of the 3rd International Workshop on Information Technologies and Systems (WITS), Orlando, Florida, USA.

Bußler, C. (1994) Enterprise Process Modeling and Enactment in GERAM. In: ICARCV (1994).

Bußler, C. and Jablonski, S. (1994) An Approach to Integrate Workflow Modeling and Organization Modeling in an Enterprise. In: Proceedings of the Third IEEE International Workshop on Enabling Technologies: Infrastructure for Collaborative Enterprises (WET ICE), Morgantown, West Virginia, USA.

Bußler, C. and Jablonski, S. (1995) Policy Resolution for Workflow Management. In: Proceedings of the Hawaii International Conference on System Sciences - 28, Maui, Hawaii.

CEN TC310 WG1 (1994) An evaluation of CIM modelling constructs - Evaluation report of constructs for views according to ENV 40 003. In: Computers in Industry. Vol. 24, Nrs.2-3.

DIISM (1993) Proceedings of the JSPE/IFIP WG 5.3 International Workshop on The Design of Information Infrastructure Systems for Manufacturing (DIISM), Tokyo, Japan, 8-10 November, 1993, Edited by H. Yoshikawa and J. Goossenaerts, Elsevier, North Holland.

Georgakapoulos, D., Hornik, M. and Shet, A. (1994) An Overview of Workflow Management: From Process Modeling to Workflow Automation Infrastructure. To appear in: Distributed and Parallel Databases. Kluwer Academic Publishers.

Goossenaerts, J. (1993) Enterprise Formulae and Information Infrastructures for Manufacturing. In: DIISM (1993).

Graefe, U. and Chan, A. (1993) An Enterprise Model as a Design Tool for Information Infrastructure. In: DIISM (1993).

ICARCV (1994) Proceedings of the International Conference on Automation, Robotics and Computer Vision (ICARCV '94), Singapore, Singapore.

IEPM (1993) Proceedings of the International Conference on Industrial Engineering and Production Management (IEPM'93), Mons, Belgium. 
IMSE (1994) Proceedings of the European Workshop on Integrated Manufacturing Systems Engineering, Grenoble, France.

Jablonski, S. (1994) MOBILE: A Modular Workflow Model and Architecture. In: Proceedings of the Fourth Working Conference on Dynamic Modelling and Information Systems (DYNMOD), Noordwijkerhout, Netherlands.

Jablonski, S. (1995) Workflow-Management-Systeme: Modellierung und Architektur. Thomson's aktuelle Tutorien, TAT 9, International Thomson Publisher.

Katzy, B.R., Eversheim, W., Dobberstein, M., Feuerborn, K., Friedrich, J., Krah, O., Müller, G. and Stepprath, F.-J. (1993) CIMOSA Pilot Implementation for Technology Transfer. In: DIISM (1993).

Marshak, R. (1993) Characteristics of a Workflow System - Mind Your P's and R's. In: Proceedings of the Groupware'93 Conference, San Jose, CA.

McCarthy, D. and Sarin, S. (1993) Workflow and Transactions in InConcert. In: Bulletin of the Technical Committee on Data Engineering, Vol. 16, No. 2.

Medina-Mores, R., Winograd, R., Flores, T. and Flores, F. (1992) The Action Workflow Approach to Workflow Management Technology. In: Proceedings of the ACM 1992 Conference on Computer Supported Cooperative Work (CSCW), Toronto, Ontario, Canada.

Mertins, K. and Jochem, R. (1993) Enterprise Integration by Modelling: Basis for Planning and Optimization of Enterprise Processes. In: IEPM (1993).

Petrie, C.J. (ed) (1992) Enterprise Integration Modeling. The MIT Press, Cambridge, Mass., USA, 1992. (Proceedings of the First International Conference on Enterprise Integration Modeling Technology (ICEIMT), Hilton Head, SC, USA.)

Schuster, H., Jablonski, S., Kirsche, T. and Bußler, C. (1994) A Client/Server Architecture for Distributed Workflow Management Systems. In: Proceedings of the Third International Conference on Parallel and Distributed Information Systems, Austin, Texas, USA.

Scheer, A.-W. and Kruse, Chr. (1994) ARIS-Framework and Toolset: A Comprehensive Business Process Reengineering Methodology. In: ICARCV (1994).

Vernadat, F. (1993) CIMOSA: Enterprise Modelling and Enterprise Integration Using a ProcessBased Approach. In: DIISM (1993).

Weston, R. and Coutts, I. (1994) Model Enactment based on use of the CIM-BIOSYS Integrating Infrastructure. In: ICARCV (1994).

WfMC (1995) Homepage of the Workflow Management Coalition. http://www.aiai.ed.ac.uk/WfMC/

Woetzel, G. and Kreifelts, T. (1993) The Use of Petri Nets for Modelling Workflow in the DOMINO System. In: Workshop on CSCW, Petri Nets and Related Formalisms, International Conference on Application and Theory of Petri Nets, June 21-23, Chicago, IL.

\section{BIOGRAPHY}

Christoph Bussler is faculty member at the Database Chair of the University of Erlangen, Germany, where is pursuing a Ph.D. degree. His research work is in two areas: workflow and organization modeling, with a focus on organizational embedding of workflow management, and architectures of execution infrastructures for enterprise-wide deployment of workflow management. While at Digital from 1991 to 1994, Christoph developed the Policy Resolution Architecture and its prototype implementation. He holds a Master of Computer Science (1990) from the Technical University of Munich and has published numerous papers on workflow management and enterprise modeling/integration. He is member of the IFIP/IFAC Task Force on Architectures for Enterprise Integration. 